\title{
THE ATTENUATION OF HIGH FREQUENCY PHONONS AT LOW TEMPERATURES*
}

\author{
R. ORBACH** and L. A. VREDEVOE*** \\ Department of Physics, University of California, Los Angeles, California 90024
}

(Received 12 June 1964)

THIS paper is concerned with the anharmonic attenuation of phonons with energies $\hbar \omega \gg k T$. Recent experiments which produce high energy phonons via nonradiative transitions in paramagnetic salts have shown [1] that at least some of these phonons have anomalously long lifetimes, corresponding to mean free paths of the order of 0.1 to $1 \mathrm{~cm}$. We have examined theoretically the attenuation of a high energy phonon at low temperatures via three and four phonon anharmonic processes. We find that, as a consequence of energy momentum conservation conditions, the lowest (transverse) branch of the phonon spectrum has an anomalously long lifetime at low temperatures, proportional to $\exp (a \hbar \omega / k T)$, where $a$ is a constant of order unity. This is in marked contrast with the much faster relaxation rates for the other two branches.

The theory for the anharmonic relaxation of phonons in the frequency region $\omega \tau_{\mathrm{th}} \gg 1$, where $\tau_{\mathrm{th}}$ is the relaxation time for phonons of energy $\approx k T$, was first given by Landau and Rumer [2]. They considered only the region $\hbar \omega \ll k T$ and their results appear to fit rather well the transverse phonon attenuation at microwave frequencies observed by Bömmel and Dransfeld [3,4]. Landau and Rumer demonstrated that low frequency $(\hbar \omega \ll k T)$ longitudinal phonons could not interact significantly with thermal phonons of energy $k T$ in a three phonon process because of energy - momentum conservation conditions [5]. We have extended their techniques to the case of phonons for which $\hbar \omega \gg k T$ and have found just the opposite: The high energy transverse phonons exhibit anomolously long lifetimes.

Conservation of energy and momentum in an isotropic solid limit three and four phonon anharmonic processes to

$$
\begin{aligned}
& t+1 \longleftrightarrow 1 \\
& t+t \longleftrightarrow 1
\end{aligned}
$$

and four phonon processes to

$$
\begin{aligned}
& 1+t \longleftrightarrow t+t \\
& 1+t \longleftrightarrow t+1 \\
& 1+t \longleftrightarrow 1+1 \\
& 1+1 \longleftrightarrow 1+1 \\
& 1+t+t \longleftrightarrow 1 \\
& 1+t+1 \longleftrightarrow 1
\end{aligned}
$$

\footnotetext{
* Supported in part by the National Science Foundation and the Office of Naval Research. ** Alfred P. Sloan Foundation Fellow. 
where $t$ designates a phonon of transverse polarization, $l$ of longitudinal polarization. We have ignored dispersion, but our results will be unaffected for $\omega$ vs $\vec{k}$ surfaces having usual convex upward curvature [6]. If $\hbar_{\omega} \gg k T$, it is clear that the dominant attenuation process will be a splitting of the high energy phonon into two phonons of energy $\sim \hbar \omega / 2$. The rate of splitting will be temperature independent. However, only longitudinal phonons may split in this manner, as can be seen from (1) and (2). It is impossible for transverse phonons to do so and still satisfy the energy and momentum conservation conditions. We have computed the three phonon longitudinal phonon lifetime for the dominant process (1a), $\tau_{1,1 t}$. We find

$$
\frac{1}{\tau_{l, 1 t}}=\frac{\hbar\left[\left(C_{1}+C_{4}\right)+\frac{3}{2}\left(C_{2}+C_{5}\right)\right]^{2}}{16 \pi \rho^{3} v_{1}{ }^{6} v_{t}{ }^{2}}\left(1-\frac{v_{t}{ }^{2}}{v_{1}{ }^{2}}\right)\left(\frac{v_{t}{ }^{2}}{v_{t}{ }^{2}}-\frac{v_{t}}{v_{1}}+\frac{3}{8}\right) \omega^{5}
$$

where the constants $C_{1}, C_{2}$, etc. are the coefficients of the deformation tensor

$$
\omega_{a \beta}=\frac{1}{2}\left\{\frac{\partial u_{\alpha}}{\partial x_{\beta}}+\frac{\partial u_{\beta}}{\partial x_{a}}+\delta_{m n}\left(\frac{\partial u_{m}}{\partial x_{a}}\right)\left(\frac{\partial u_{n}}{\partial x_{\beta}}\right)\right\},
$$

in the expression for the elastic energy

$$
\begin{aligned}
\mathscr{H}=\frac{1}{2} C_{1}\left(\omega_{\alpha \alpha}\right)^{2}+\frac{1}{2} C_{2}\left(\omega_{\alpha \beta}\right)^{2} & +\frac{1}{2} C_{3}\left(\omega_{\alpha \alpha}\right)^{3}+\frac{1}{2} C_{4} \omega_{\alpha \alpha}\left(\omega_{\beta \gamma}\right)^{2}+\frac{1}{2} C_{5}\left(\omega_{\alpha \beta}\right)^{3}+\frac{1}{2} C_{6}\left(\omega_{\alpha \alpha}\right)^{4} \\
+ & \frac{1}{2} C_{7} \omega_{\alpha \alpha}\left(\omega_{\beta \gamma}\right)^{3}+\frac{1}{2} C_{8}\left(\omega_{\alpha \alpha}\right)^{2}\left(\omega_{\alpha \beta}\right)^{2}+\frac{1}{2} C_{9}\left(\omega_{\alpha \beta}\right)^{4}+\frac{1}{2} C_{10}\left(\omega_{\alpha \beta}\right)^{2}\left(\omega_{\alpha \beta}\right)^{2} .
\end{aligned}
$$

Here, $u_{a}$ is the displacement from equilibrium in the $a$ direction, one sums over repeated indeces, and $\omega_{\alpha a}=\operatorname{Tr} \omega_{\alpha \beta},\left(\omega_{\alpha \beta}\right)^{2}=\operatorname{Tr} \omega_{\alpha \beta} \omega_{\beta \gamma}$, etc. Equation (3), for the representative values, $v_{1}=5 \times 10^{5}$ $\mathrm{cm} / \mathrm{sec}, v_{t}=v_{1} / \sqrt{3}, \rho=3 \mathrm{gr} / \mathrm{cm}^{3}, C=10^{12}$ dyne $-\mathrm{cm}^{-2}$, yields a lifetime of $5 \times 10^{-8} \mathrm{sec}$ for a phonon with energy $30 \mathrm{~cm}^{-1}$, or a mean free path of $250 \mu$.

Expressions (1) and (2) show that it is impossible for a transverse phonon to decay by splitting into two phonons. However, one might expect that a transverse phonon could combine with another phonon of energy $\sim k T$ (via processes (1a) or (1b)) to form a longitudinal phonon which would then decay rapidly. A simple argument shows that this is impossible if $\hbar_{\omega} \gg k T$. Labeling the high energy phonon by wave vector $\vec{k}_{1}$, the thermal phonon of energy $\sim k T$ by wave vector $\vec{k}_{2}$, the angle between $\vec{k}_{1}$ and $\vec{k}_{2}$ by $\theta_{12}$, energy and momentum conservation result in the equations

$$
\begin{aligned}
& \text { Process (1a) } \quad \frac{\left|\vec{k}_{1}\right|}{\left|\vec{k}_{2}\right|}=\frac{1-\cos \theta_{12}}{1-\left(v_{t} / v_{1}\right)}, \\
& \text { Process (1b) } \quad \frac{\left|\vec{k}_{1}\right|}{\left|\vec{k}_{2}\right|}=\frac{1-\left(v_{1} / v_{t}\right) \cos \theta_{12}}{1-\left(v_{t} / v_{1}\right)} .
\end{aligned}
$$

By construction $\hbar \omega \gg k T$ so that $\left|\vec{k}_{1}\right| \gg\left|\vec{k}_{2}\right|$, and there exist no values of $\theta_{12}$ which can satisfy Eqs. (4a), (4b). It is necessary to use a longitudinal or transverse phonon in processes (1a) or (1b) respectively, of energy $\sim \hbar \omega$ to relax the high energy transverse phonon. This will reduce the relaxation rate by a factor $\exp (\hbar \omega / k T)$, a number which can be made arbitrarily large at sufficiently low temperatures. In detail, we find for temperatures $T \ll \frac{\hbar \omega}{k}$,

Process (1a)

$$
\begin{aligned}
\frac{1}{\tau_{t 1,1}}=\frac{\hbar\left[\left(C_{1}+C_{4}\right)+\frac{3}{2}\left(C_{2}+C_{5}\right)\right]^{2}}{32 \pi \rho^{3} v_{1}{ }^{7} v_{t}^{2}}\left[1-\left(\frac{v_{t}}{v_{l}}\right)^{2}\right] \omega^{3}\left(\frac{k T}{\hbar}\right)^{2} & \\
& \times \exp \left[-\frac{\hbar \omega}{2 k T}\left(\frac{v_{1}-v_{t}}{v_{t}}\right)\right],
\end{aligned}
$$


Process (1b) $\quad \frac{1}{\tau_{t t, 1}}=\frac{(1.17) \hbar\left[2 C_{2}+C_{4}+3 C_{5}\right]^{2}\left(1-v_{t} / v_{l}\right)^{3}}{384 \rho^{3} \pi v_{t}{ }^{7} v_{1}^{2}\left(1+v_{t} / v_{1}\right)^{4}} \omega^{5} \exp \left[-\frac{\hbar \omega}{k T}\left(\frac{1-\left(v_{t} / v_{l}\right)}{1+\left(v_{t} / v_{1}\right)}\right)\right]$.

For a transverse phonon of energy $\sim 30 \mathrm{~cm}^{-1}$ at $1^{\circ} \mathrm{K}$ we find

$$
\frac{1}{\tau} \cong \frac{1}{\tau_{t, t}}=1.4 \times 10^{6} \exp (-11) \mathrm{sec}^{-1}=26 \mathrm{sec}^{-1}
$$

or a relaxation time six orders of magnitude longer than for a longitudinal phonon of the same energy at the same temperature. To see if four phonon anharmonic processes could change this result we have investigated the allowed processes in (2). We find as a representative result,

$$
\frac{1}{\tau_{t 1,11}} \cong \frac{\left(3.4 \times 10^{-2}\right) \hbar^{2} C_{\text {eff }}^{2}}{(2 \pi)^{5} \rho^{4} v_{1}^{12} v_{t}^{2}} \omega^{6}\left(\frac{k T}{\hbar}\right)^{3},
$$

where $C_{\text {eff }}$ is a combination of the coefficients $C_{1}, C_{2}, \ldots, C_{10}$ in Eq. (4). After suitable angular averages,

$$
C_{\text {eff }}^{2}=16\left\{8\left(C_{4}+C_{7}\right)^{2}+\frac{2}{3}\left(3 C_{3}+2 C_{8}\right)^{2}+\frac{64}{27}\left(C_{2}+4 C_{5}+4 C_{9}\right)^{2}+\frac{4}{15}\left(C_{1}+4 C_{4}+4 C_{10}\right)^{2}\right\} .
$$

Again, we let $C_{i} \sim 10^{12}$ dyne $\mathrm{cm}^{-2}$ to find $C_{\text {eff }}{ }^{2}=4 \times 10^{27} \mathrm{dyne}^{2} \mathrm{~cm}^{-4}$. The fourth order relaxation rate for a phonon of energy $30 \mathrm{~cm}^{-1}$ at $1^{\circ} \mathrm{K}$ is then found to be

$$
\frac{1}{\tau_{t 1,11}} \cong 10^{-3} \sec ^{-1}
$$

much too small to be significant. Lack of isotropy in a real crystal which allows two different transverse branches propagating near a degeneracy direction to interact [5] is also negligible. Umklapp can be neglected as long as $\omega / v \ll k_{\mathrm{D}}$ since the selection rules Eqs. (1) and (2) are unaltered.

The long lifetime of the lowest branch of the phonon spectrum for phonon energies $\gg k T$ raises the possibility of generating "hot" phonons at very low temperatures. There appears to be some evidence for this already in the experiments of Geschwind [1] who has observed an apparent heating of the $30 \mathrm{~cm}^{-1}$ phonons in ruby due to the nonradiative relaxation of the upper ${ }^{2} E$ state. The long transverse phonon lifetime also makes possible the detection of these phonons by neutron diffraction, as has been suggested by Blume and Geschwind [7]. The difference in lifetime between longitudinal and transverse phonons of high energy at low temperatures may also be observable in phonon assisted tunneling experiments. One would expect the peak in the tunneling current associated with the transverse phonons to be sharper than the peak associated with the longitudinal phonon branch because of the former's longer intrinsic lifetime. Virtual processes which involve the exchange of high energy phonons may also be influenced since the contribution of the imaginary part of the energy denomi nator will be larger for longitudinal than for transverse phonons.

We wish to thank Dr. Geschwind for the initial impetus for this investigation and for allowing us to refer to his results prior to their publication. We also wish to thank Dr. C. Herring for some helpful comments.

\section{References}

1. S. GESCHWIND, private communication.

2. L. LANDAU and G. RUMER, Phys. Zeit. Sowjetunion 11, 18 (1937); S. SIMONS, Proc. Camb. Phil. Soc. 53, 702 (1957).

3. H. BOMMEL and K. DRANSFElD, Phys. Rev. 117, 1245 (1960).

4. R. ORBACH, Thesis, University of California, Berkeley, California, 1960. Copies are available from the author.

5. C. HERRING, Phys. Rev. 95, 954 (1954) demonstrated that lack of isotropy in real crystals can allow a longitudinal phonon to interact with the two different transverse branches propagating near a degeneracy direction. Subsequent numerical estimates [4] have shown this process to be relatively unimportant for long wavelength phonons at low temperature. Simons (Proc. Phys. Soc. 82, 401 (1963)) has pointed out 
that the existence of a finite phonon relaxation time will in fact allow three phonon relaxation of longitudinal phonons. Such effects will be unimportant in the frequency region $\hbar \omega \gg k T$ because the dispersion is much too great for the phonon width to make up the energy difference $\approx \hbar \omega(2 \vec{k})-2 \hbar \omega(\vec{k})$.

6. R. PEIERLS, Quantum Theory of Solids, Oxford University Press, 1956, page 44.

7. M. BLUME and S. GESCHWIND, private communication. 